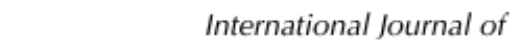 \\ Applied
Ceramic \\ TECHNOLOGY
}

Ceramic Product Development and Commercialization

\section{Crystallographic Characteristics of Grain Boundaries in Dense Yttria-Stabilized Zirconia}

\section{Lam Helmick}

National Energy Technology Laboratory, Morgantown, West Virginia 26507

Department of Materials Science and Engineering, Carnegie Mellon University, Pittsburgh, Pennsylvania 15213

\section{Shen J. Dillon}

Department of Materials Science and Engineering, University of Illinois at Urbana-Champaign, Urbana, Illinois 61801

\section{Kirk Gerdes and Randall Gemmen}

National Energy Technology Laboratory, Morgantown, West Virginia 26507

\section{Gregory S. Rohrer}

Department of Materials Science and Engineering, Carnegie Mellon University, Pittsburgh, Pennsylvania 15213

\section{Sridhar Seetharaman and Paul A. Salvador ${ }^{\star}$}

National Energy Technology Laboratory, Morgantown, West Virginia 26507

Department of Materials Science and Engineering, Carnegie Mellon University, Pittsburgh, Pennsylvania 15213

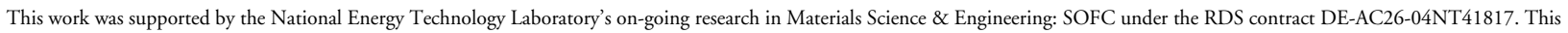
work was partially supported by the MRSEC program of the National Science Foundation under Award Number DMR-0520425 and by the Pennsylvania DCED. 
Grain-boundary plane, misorientation angle, grain size, and grain-boundary energy distributions were quantified using electron backscatter diffraction data for dense polycrystalline yttria-stabilized zirconia, to understand interfacial crystallography in solid oxide fuel cells. Tape-cast samples were sintered at $1450^{\circ} \mathrm{C}$ for $4 \mathrm{~h}$ and annealed for at least $100 \mathrm{~h}$ between $800^{\circ} \mathrm{C}$ and $1650^{\circ} \mathrm{C}$. Distributions obtained from both three-dimensional (3D) reconstructions and stereological analyses of $2 \mathrm{D}$ sections demonstrated that the (100) boundary planes $\{(111)\}$ have relative areas larger $\{$ smaller $\}$ than expected in a random distribution, and that the boundary plane distribution is inversely correlated to the boundary energy distribution.

\section{Introduction}

Solid oxide fuel cells (SOFCs) are attractive energy technologies because they can efficiently convert a range of fuels to electricity. ${ }^{1}$ The performance of a SOFC is coupled strongly to microstructural features, which have now been nearly optimized with respect to traditional metrics, such as grain size, volume fraction, and connectivity of all phases. ${ }^{1-4}$ However, improvements in performance are still sought to decrease both the level of loss in initial cells and the degradation of cells versus time. Recent advances in characterization techniques have allowed for the direct three-dimensional (3D) reconstruction of the microstructure of SOFC electrodes and for correlations to be made directly between microstructural features, such as the length of the triple phase boundary lines, and the performance of electrodes. $^{5-8}$ It is also possible to include the crystallographic nature of internal interfaces or surfaces, ${ }^{9-14}$ which are known to affect properties of polycrystals, ${ }^{15}$ including transport, ${ }^{16-20}$ electrochemistry, and corrosion, ${ }^{15,21}$ but these characteristics have not yet been included in SOFC characterization. ${ }^{22-24}$ The crystallographic nature of internal interfaces can be measured using ex situ 3D reconstruction methods for operational SOFCs, ${ }^{13,14,25}$ but several challenges still exist to interpreting and understanding the significance of the distributions of crystallographic features in such complex microstructures. These challenges include the lack of information on the crystallographic distributions in the relevant material systems, such as for yttria-stabilized zirconia, their time evolution with respect to temperature and electrochemistry, and effects of other phases on these distributions. In the long run, understanding the driving forces for microstructural changes and the properties of specific crystallographic features will help to understand the long-term evolution and performance of SOFC components. In this paper, as a step toward understanding the more complex cathode and anode of SOFCs, we report on the grain-boundary plane normal, misorientation angle, grain size, and grain-boundary energy distributions (GBEDs) in dense polycrystalline yttria-stabilized zirconia (YSZ) that were prepared following representative planar SOFC fabrication methods.

It is increasingly clear that polycrystalline materials have anisotropic grain-boundary character distributions (GBCDs); ${ }^{9,11,12,14,25-30}$ the GBCD, or $\lambda(\Delta g, n)$, is the quantitative five-parameter description of the relative amounts of boundaries with respect to lattice misorientation, $\Delta g$, and grain-boundary plane normal, $n$. Using automated collection and analysis of electron backscatter diffraction (EBSD) patterns, the GBCDs of variety of materials have been measured, including the ceramics $\mathrm{MgO},{ }^{12,30,31} \mathrm{SrTiO}_{3},{ }^{11} \mathrm{Y}_{2} \mathrm{O}_{3},{ }^{13} \mathrm{Al}_{2} \mathrm{O}_{3},{ }^{32}$ $\mathrm{MgAl}_{2} \mathrm{O}_{4},{ }^{10,33} \mathrm{TiO}_{2},{ }^{10}$ and yttrium aluminum garnet. ${ }^{32}$ For these materials, it has been observed that the GBCD develops to a steady-state value for which an inverse correlation exists between the populations of specific boundaries and the energies of those boundaries, or between the GBCD and the GBED. Simulations and experiments also have provided an insight into the development of anisotropic GBCDs during normal grain growth owing to anisotropies in GBED, 9,10,28,29,32,34,35 although only a few GBEDs have been measured owing to the experimental challenges. Although aspects of the GBEDs have been rationalized using the anisotropies of surface energies (speculations that cannot be used to rationalize our observations on YSZ), ${ }^{10,13}$ no general theory is available to predict or compute the GBED of a general polycrystal. As such, it is essential to measure GBCDs and GBEDs of important technological materials, for which it is appropriate to prepare samples in manners similar to those used in technology. Despite the technological importance of YSZ, especially in SOFCs, no reports of the GBCDs or GBEDs exist for it or for other fluorite compounds. We are particularly interested in determining the steady-state distributions, because any parameter that is correlated to microstructural features will no longer evolve owing to changes in the relative boundary populations. 
YSZ has the cubic fluorite structure ${ }^{36}$ and has long been a principle functional material for SOFCs. ${ }^{2}$ The high ionic conductivity, wide stability range versus oxygen pressure, and low chemical reactivity of YSZ are key features to its use in conventional SOFCs, foremost not only as the dense electrolyte but also as the ion conducting phase in both active electrodes. ${ }^{2}$ There have been various studies on the microstructure of YSZ polycrystals, focusing on grain size, ${ }^{37}$ pore morphology, ${ }^{38}$ and pore distribution. ${ }^{39}$ Yet, there is little known about the crystallographic features present at grain boundaries or other internal interfaces. Because the GBCD controls properties and microstructural evolution, it has important implications for long-term activity of electrodes in SOFCs, both for performance and degradation.

As a step toward understanding these two essential features of SOFC performance, we investigated dense YSZ compacts to determine features of the GBCD especially the grain size, grain-boundary plane distribution, and misorientation angle distribution. For most samples, distributions were determined from stereological analyses of 2D EBSD maps, owing to its relative simplicity. For one YSZ sample, a 3D orientation map of the microstructure was also made from a series of parallel EBSD maps collected using a dual-beam focused ion beam scanning electron microscope (FIB-SEM). ${ }^{13,14}$ After reconstructing the 3D microstructure in a manner similar to that carried out previously, ${ }^{14}$ these data were used to calculate the grain-boundary plane normal distributions directly, as well as the GBED to understand the nature of the distribution, which are also compared with computations of the surface energy anisotropy available in the literature.

\section{Experimental Procedure}

\section{Sample Preparation}

A YSZ (zirconia doped with $8 \%$ yttria, according to the composition $\left.\mathrm{Y}_{0.16} \mathrm{Zr}_{0.84} \mathrm{O}_{2-\delta}\right)$ slurry was prepared using methods modified from those given in Mistler and Twiname $^{40}$ (and detailed in the supporting information) using YSZ powder (YSZ8-U1, fuelcellsmaterials.com, 300-500 nm particle size, NexTech Materials, Lewis Center, $\mathrm{OH})$. The 127-mm-thick tapes were cast, dried, and punched to form cylindrical coupons. Assintered (AS) samples were formed by compressing stacks of 12-16 coupons uniaxially and sintering them at $1450^{\circ} \mathrm{C}$ for $4 \mathrm{~h}$. Different AS samples were individually annealed for $672 \mathrm{~h}$ at $800^{\circ} \mathrm{C}$ or for $100 \mathrm{~h}$ at $1250^{\circ} \mathrm{C}, 1450^{\circ} \mathrm{C}$, and $1650^{\circ} \mathrm{C}$, called respectively the $800,1250,1450$, and 1650 samples.

Samples were prepared for 2D OIM by polishing with $0.02 \mu \mathrm{m}$ colloidal slurry and then annealing them at $1000^{\circ} \mathrm{C}$ for $1 \mathrm{~h}$. The sample for 3D FIB-SEM was prepared as described previously. ${ }^{14}$ Triangular samples were prepared mechanically, sputter-coated with $\sim 2 \mathrm{~nm}$ of platinum, and attached onto $45^{\circ}$ pretilted stubs. The specimen tips were ion milled to create a large flat scan area for EBSD. Circular fiducial marks were milled into the samples using the ion beam in the FIB (Nova 600, FEI, Hillsboro, OR) to align the area during subsequent milling and EBSD mapping steps. ${ }^{13,14,25}$

\section{EBSD Data Collection and Cleanup}

The local orientation of all samples was recorded at discrete points on a hexagonal grid using EBSD. Stereological data were obtained (except the 1650 sample) using a grid spacing of $0.25 \mu \mathrm{m}(2.5 \mu \mathrm{m}$ for $1650 \mathrm{sam}$ ple). ${ }^{41}$ Two-dimensional EBSD data were collected in an environmental SEM (Quanta 200, FEI) using a $25 \mathrm{kV}$ beam with a spot size of 5.0. For the 3D FIBSEM, data were collected as described previously. ${ }^{13,14}$ Briefly, the sample was ion milled at $30 \mathrm{kV}$ and $3 \mathrm{nA}$ using a Ga${ }^{+}$ion beam. EBSD data were acquired using a $30 \mathrm{kV}$ beam at a current of $9.5 \mathrm{nA}$. The in-plane step size was $0.2 \mu \mathrm{m}$ and the out-of-plane step size was $0.4 \mu \mathrm{m}$. In both the $2 \mathrm{D}$ and $3 \mathrm{D}$ cases, multiple data sets were collected and merged to generate sufficient observations to make statistical comparisons.

The EBSD data were processed using the commercial TSL OIM Analysis software, first using two iterations of grain dilation (a minimum grain size of 10 pixels and a grain tolerance angle of $5^{\circ}$ ), then assigning a single average orientation to each grain (minimum grain size of 5 pixels), and finally removing points not associated with grains. In the $2 \mathrm{D}$ data, grain boundaries were reconstructed with a maximum deviation of 2 pixels, using a procedure described by Wright and Larsen. ${ }^{42}$

For the 3D FIB-SEM reconstruction, the processed data were aligned using commercially available 3D OIM visualization software (EDAX), and line segments were processed using a program written in-house. ${ }^{13,14,25,26}$ Triple points on two parallel layers were connected by a 
triple line when the orientations between the three grains around the first junction and the three grains around one of the five closest junctions were within $5^{\circ} .{ }^{13}$ Only grain-boundary segments adjacent to triple junctions were analyzed, and the grain-boundary plane normals were found following a procedure described elsewhere. ${ }^{13}$ Errors associated with the 3D data collection are discussed elsewhere, ${ }^{11-13,30}$ and a secondary alignment process ${ }^{13}$ was carried out to minimize errors arising from uncertainty in the horizontal alignment of the layers during reconstruction. 3D orientation maps were created by assigning identification numbers to grains in individual layers, calculating the mean position of each grain, and then comparing grain orientations from each layer to adjacent layers. If grains from adjacent layers had a disorientation $<3^{\circ}$ and a mean position separated by less than two mean grain diameters, they were assigned the same grain identification number.

\section{EBSD Data Analysis}

The average grain size (determined from the grain area $^{43}$ and reported as the diameter of a circle of equivalent area) and grain size distribution were determined from at least 6800 grains. The reconstructed grainboundary segments and the crystal orientations associated with them were used to extract portions of the overall GBCD, $\lambda(\Delta g, n)$. The misorientation $\Delta g$ was analyzed using the common axis $(l)$ and angle of rotation $(\theta)$ approach. Plots of the number fraction of segments having a misorientation angle $\theta$, independent of $l$ and $n$, were generated using TSL OIM Analysis software. The misorientation averaged distribution of grainboundary plane normals, $\lambda(\mathrm{n})$ or the grain-boundary plane distribution (GBPD), was determined using an inhouse program (G.S. Rohrer, personal communication) that indicates the relative areas of different grain-boundary plane orientations. The relative areas of all orientations were normalized to yield values that are equivalent to multiples of a random distribution (MRD); in these units, values that differ from unity indicate orientations that have areas greater than or less than that expected in a random, isotropic distribution. As carried out elsewhere, the misorientation averaged boundary plane distributions were classified into bins of $10^{\circ}$ and plotted on a stereographic projection, resulting in approximately 13 discrete orientations within the cubic stereographic triangle. $^{11,12}$ Each stereological distribution was deter- mined using at least 20,000 boundary segments to provide statistical significance to the GPBDs. ${ }^{41}$

For the 3D reconstruction, the GBCD was calculated from the extracted triangles using the methods described previously. ${ }^{30}$ Briefly, the five-parameter space is discretized into equal volume bins spanning approximately $10^{\circ}$ in each parameter. Each triangular area was placed in the bin corresponding to its misorientation and boundary orientation, and all of the symmetrically equivalent bins. There were 170,000 triangles and $99.3 \%$ of the bins in the discretized GBCD contained at least 10 triangles. The 5D data, which will be further analyzed and discussed elsewhere, were averaged over other variables to generate a misorientation averaged GBPD, to compare with the stereological data. ${ }^{13}$

To determine whether the distributions were significantly different from random distributions, ${ }^{41}$ a randomized data set was generated using the processed data of YSZ samples. In the randomization procedure, the Euler angles (used to parameterize orientations) were scrambled for the two grains associated with each reconstructed boundary segment. The randomized data were then processed in the same fashion as the actual experimental data to generate randomized GBPDs that were directly comparable with the experimental GBPDs.

The GBED was calculated using the method described by elsewhere. ${ }^{44} 58,000$ triple junctions were included in the data set and the calculation used a relaxation factor of 0.05 . The calculation was assumed to have converged after adjustments to the capillarity vector field were $<1 \%$ of the initial adjustment. Finally, the GBED was smoothed by averaging the capillarity vectors in neighboring bins. ${ }^{30}$ Again, the GBED was averaged over misorientation to generate a GBED that could be compared with the GBPD.

\section{Results}

Orientation maps from a representative region of an AS dense YSZ sample are given in Fig. 1. The as-collected data are shown in Fig. 1a and the processed data are shown in Fig. 1b, which include reconstructed boundary segments. Figure 1a illustrates that the ascollected data were well indexed (there are relatively few pixels that are of random orientations relative to their neighbors). This was true for all regions of all samples discussed in this work. The average confidence index, a 

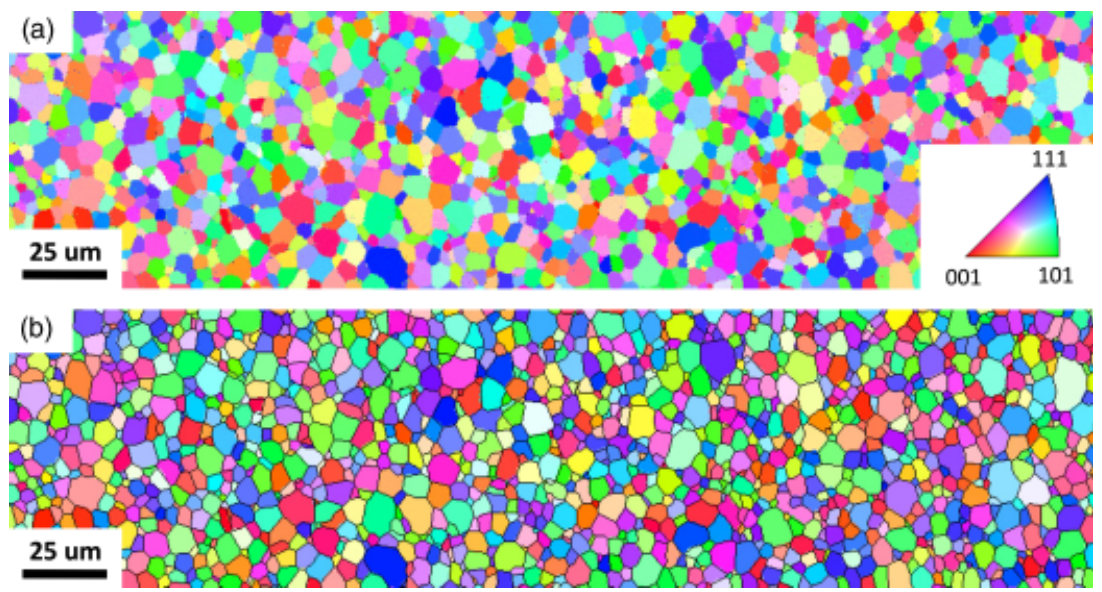

Fig. 1. Inverse pole figure map of a representative region of as-sintered sample, where (a) are the as-collected data and (b) are the cleaned data with reconstructed boundaries.

measure of the quality of the EBSD pattern and the accuracy of the assignment of an orientation based on the EBSD pattern, ${ }^{43}$ was at least 0.61 for all samples, which greatly exceeds the minimum confidence index of 0.1 recommended by EDAX. ${ }^{43}$ By visual comparison of Figs. 1a and b, it is clear that the data processing yields an accurate representation of the grain orientations, grain sizes, and grain-boundary planes. This was true for all samples discussed herein.

These tape-cast YSZ samples, which were produced in a manner typical to SOFC production, exhibited equiaxed grains having no significant orientation texture, both of which are evident in the representative region shown in Fig. 1. The average grain sizes (diameter) for the different samples are reported in Table I and the grain size distributions, in area fraction, for all samples are given Fig. 2. The AS, 850, 1250, and 1450 samples had an average grain size of $5( \pm 2) \mu \mathrm{m}$, with $6-8 \mu \mathrm{m}$ grains dominating the area fraction population. The

Table I. Average Grain Diameters for Various Annealing Temperatures (and Time)

\begin{tabular}{lcc}
\hline $\begin{array}{l}\text { Temperature } \\
\left({ }^{\circ} \mathbf{C}\right)\end{array}$ & Time $(\mathbf{h})$ & $\begin{array}{c}\text { Average grain } \\
\text { diameter }(\boldsymbol{\mu m})\end{array}$ \\
\hline 1450 as-sintered & 4 & $5( \pm 2)$ \\
1450 & 100 & $5( \pm 2)$ \\
800 & 672 & $5( \pm 2)$ \\
1250 & 100 & $5( \pm 2)$ \\
1650 & 100 & $60( \pm 30)$ \\
\hline
\end{tabular}

1650 sample had an average grain size of $60( \pm 30) \mu \mathrm{m}$ with most prevalent grain sizes between 80 and $90 \mu \mathrm{m}$.

All samples exhibited misorientation angle distributions that were consistent with random distributions. Figure 3 shows the misorientation angle distribution of the AS sample, where the blue line represents the YSZ distribution and the red line represents the Mackenzie (random) distribution. ${ }^{45,46}$ The $800,1250,1450$, and 1650 samples had similar distributions to the AS sample. No significant deviations from the random distribution could be observed.

The grain-boundary plane distributions, $\lambda(\mathrm{n})$, of the AS $\left(1450^{\circ} \mathrm{C}\right.$ for $\left.4 \mathrm{~h}\right)$ and the 1450 (AS $+1450^{\circ} \mathrm{C}$ for $100 \mathrm{~h}$ ) samples are shown in Figs. $4 \mathrm{a}$ and b, respectively. The GBPDs are plotted in standard stereographic

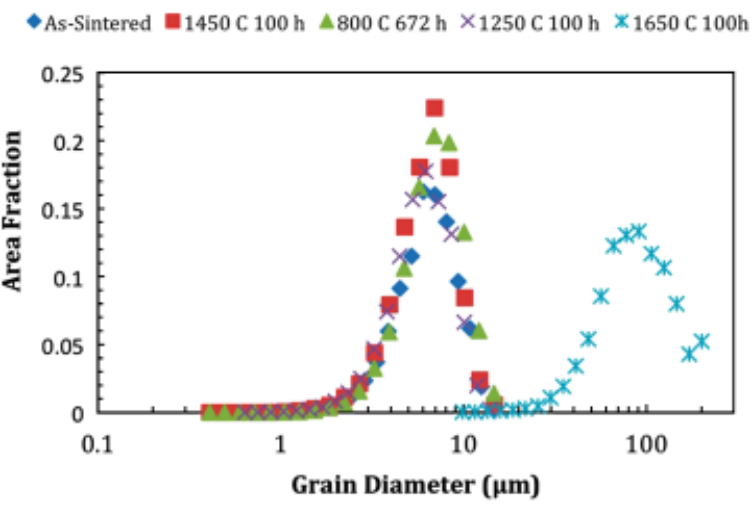

Fig. 2. Grain size distributions for the as-sintered, 1450, 800, 1250 , and 1650 samples. 


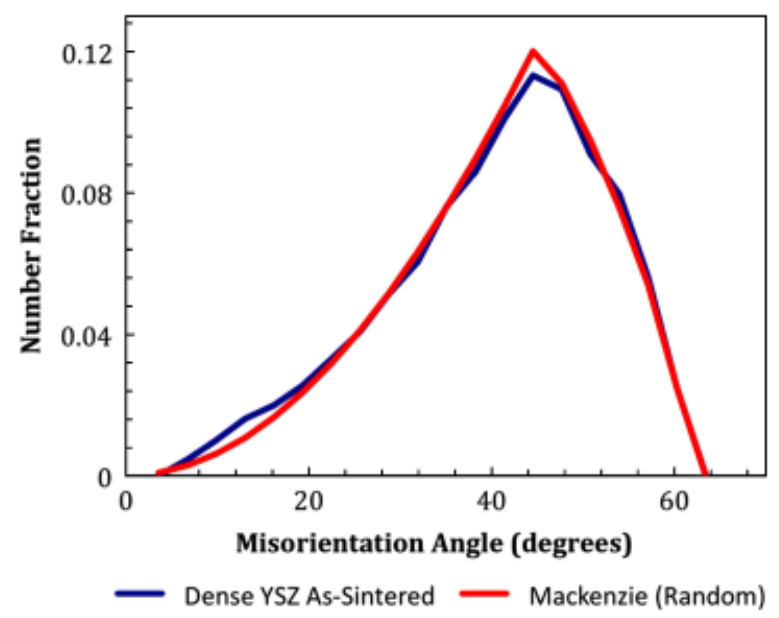

Fig. 3. Misorientation angle distribution of as-sintered sample.

triangles for a cubic material. The color key for all plots is given at the bottom of Fig. 4. Below each distribution, the range of the MRD observed in the triangle and the number of boundary segments used to construct the GBPD are given. Not surprisingly (because the longer anneal at $1450^{\circ} \mathrm{C}$ did not lead to an increase in the grain

(a) $1450^{\circ} \mathrm{C}, 4 \mathrm{~h}$

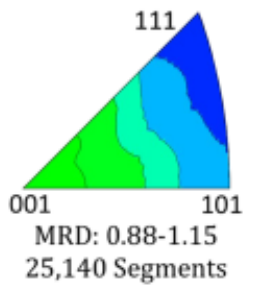

(c) $1450^{\circ} \mathrm{C}, 4 \mathrm{~h}$ Randomized

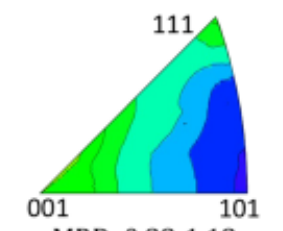

MRD: $0.88-1.18$

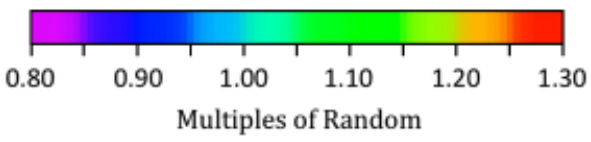

Fig. 4. Grain-boundary plane distributions for experimental data of the (a) as-sintered (AS) and (b) 1450 samples, and for the randomized data of the (c) AS and (d) 1450 samples. MRD, multiples of a random distribution. size), the two distributions are similar to one another. The samples exhibit weak anisotropy in their GBPDs, with (111) planes occurring the least frequently and the (001) planes occurring the most frequently. It should be noted that the range of the MRD values is relatively narrow-the largest being $0.84-1.19$. This distribution is among the most narrow observed for many ceramics, ${ }^{13}$ indicating that YSZ prepared in a fashion appropriate for SOFC technologies exhibits some of the weakest anisotropy in the GPBDs of ceramics known to date.

The randomized GBPDs for the AS and 1450 samples are shown in Figs. 4c and d, respectively. The limits of these randomized distributions are similar to those of the experimental distributions, ranging between 0.88 and 1.18 MRD. However, the maximum and minimum of the MRD values in the two randomized distributions are different from one another, as well as to those locations in the experimental GBPDs. These observations indicate that the anisotropies observed in the GBPDs of YSZ are experimentally significant, albeit mild in extent. In particular, the decreased frequency of (111) observations in the experimental data is distinct from that in the randomized data.

The GBPDs are plotted in standard stereographic triangles for the 800, 1250, and 1650 samples in Figs. $5 \mathrm{a}-\mathrm{c}$, respectively. The color key for all plots is given at the bottom of Fig. 5. Below each distribution, the range of values observed in the triangle and the number of boundary segments used to construct the GBPDs are given. Qualitatively, all the GPBDs are similar to those for the AS and 1450 samples, shown in Figs. 4a and b. All samples exhibit weak anisotropy in their GBPDs, with (111) planes occurring the least frequently and the (001) planes occurring the most frequently. The width of the distribution is relatively narrow-the largest being $0.83-1.19$. Because the grain sizes of the 800 and 1250 samples were similar, the similarity of the GPBDs is unsurprising and small differences likely represent the uncertainties associated with experiment. The 1650 sample had an order of magnitude increase in the grain size compared with the other samples, but no significant difference can be observed in the GBPD, indicating that the steady-state grain size distribution is obtained during the initial sintering step.

The data for these three samples were subjected to the randomization process and then reprocessed to generate new GBPDs. The GBPDs generated from the randomized data for the 800,1250 , and 1650 samples 
(a) $800^{\circ} \mathrm{C}, 672 \mathrm{~h}$

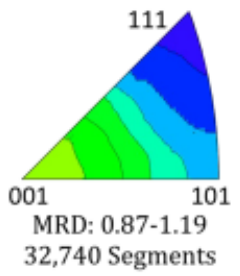

(d) $800^{\circ} \mathrm{C}, 672 \mathrm{~h}$ Randomized

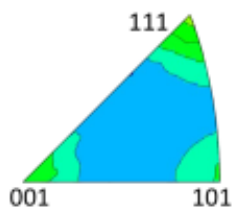

MRD: $0.93-1.27$ (b) $1250^{\circ} \mathrm{C}, 100 \mathrm{~h}$

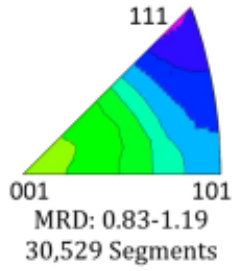

(e) $1250^{\circ} \mathrm{C}, 100 \mathrm{~h}$ Randomized

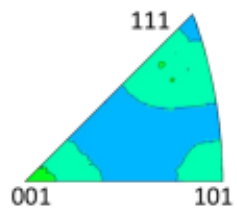

MRD: $0.92-1.08$ (c) $1650^{\circ} \mathrm{C}, 100 \mathrm{~h}$

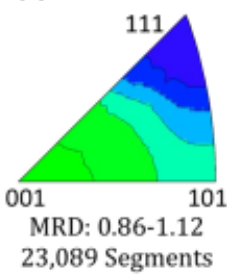

(f) $1650^{\circ} \mathrm{C}, 100 \mathrm{~h}$ Randomized

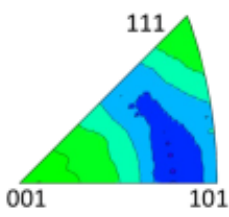

MRD: $0.86-1.16$

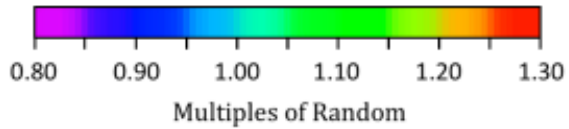

Fig. 5. Grain-boundary plane distributions for the experimental data of the (a) 800, (b) 1250, and (c) 1650 samples, and for the randomized data of the (d) 800, (e) 1250, and (f) 1650 samples. $M R D$, multiples of a random distribution.

are given in Figs. 5d-f, respectively. Because the location of maxima and minima in the GBPDs of the randomized data differ from one another and from those of the experimental GBPDs, the weak anisotropy in the real data is considered significant. Again, the most consistent difference between the distributions from the actual and randomized data is that the relative area of the (111) planes in the distributions from the actual data is lower than those in the randomized data.

The results from the 3D FIB-SEM are depicted in Figs. 6 and 7, registered on the AS sample. The 3D orientation map is shown in Fig. 6. The data collection generated high-quality EBSD patterns that cleaned up well, leaving few discarded pixels and high-quality reconstructed grain boundaries. The grain structure was equiaxed and the average grain size was in agreement with the grain size from the 2D data. As discussed elsewhere, visualization of individual grains also indicated that the grains were equiaxed. ${ }^{14}$ The misorientation angle distribution from the $3 \mathrm{D}$ data is also consistent with random grain orientation distribution.

The misorientation averaged GBPD is shown in Fig. $7 \mathrm{a}$ in stereographic projection along the (001) pole of a cubic system. Qualitatively, this distribution (from

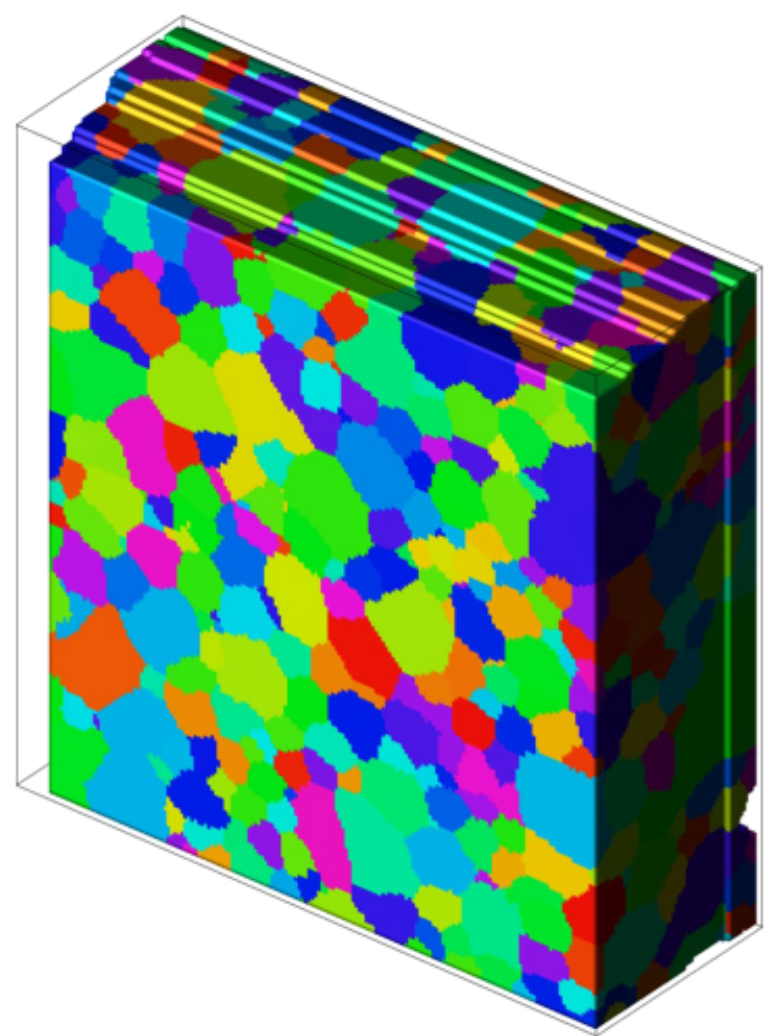

Fig. 6. Three-dimensional orientation map of YSZ for the assintered sample.

170,000 triple lines) is similar to the stereological distributions shown in Fig. 4 (and Fig. 5). A weak anisotropy exists in the $3 \mathrm{D}$ GBPD, with (111) planes occurring the least frequently and the (001) planes occurring the most frequently. The width of the distribution is even more narrow than that observed in the stereological data-the range being from 0.94 to 1.12 MRD (compared with 0.88-1.15 MRD). Again, randomizing the boundary segments indicated that the distribution was nonrandom and that the weak anisotropy was experimentally significant. The randomized result is shown in Fig. 7c. In general, GBCDs generated from $3 \mathrm{D}$ data underestimate the anisotropy in comparison with GBCDs generated from stereological approaches, although the agreement here is reasonable. ${ }^{13}$

The misorientation averaged GBED is shown in Fig. $7 \mathrm{~b}$ in stereographic projection (using the full projection) along the (001) pole of a cubic system. Here, the color scale indicates the normalized grain-boundary energy, in arbitrary units, such that large values indicate 
(a) GBPD

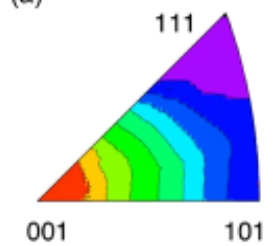

(c) GBPD

randomized
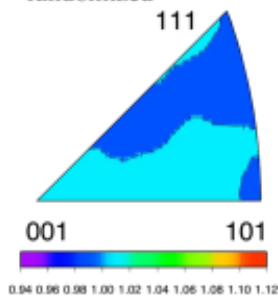

Multiples of Random (b) GBED

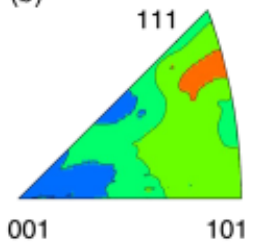

(d) GBED

randomized

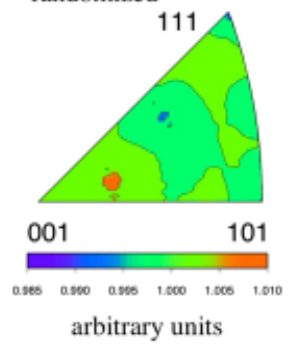

Fig. 7. The misorientation averaged grain-boundary plane distribution (GBPD) (a) and grain-boundary energy distribution (GBED) (b), as well as the randomized GBPD (c) and GBED (d), are given as stereographic projections for the as-sintered sample.

a relatively high-energy grain boundary and small values indicate a relatively low-energy boundary. The range in anisotropy in the averaged grain-boundary energy values is from 0.985 to 1.025 , which is similar to that observed for $\mathrm{Y}_{2} \mathrm{O}_{3} .{ }^{13}$ More importantly, there is an inverse correlation between the GBPD and the GBED, where high-energy grain boundaries occur less frequently in the overall population than do low-energy planes. Figure $7 \mathrm{~d}$ gives the same GBED generated from the randomized data, indicating that the variation in energy is significant.

\section{Discussion}

The GBPDs of dense polycrystalline tape-cast YSZ were found to be weakly anisotropic, with a decreased preference for (111) habit planes and an increased preference for (100) habits. The misorientation angle distribution was found to be isotropic, for all samples. The magnitude of the anisotropy in the GBPD, which ranged from 0.83 to $1.19 \mathrm{MRD}$ in the $2 \mathrm{D}$ data sets and $0.94-1.12 \mathrm{MRD}$ in the $3 \mathrm{D}$ set, is generally small in comparison with that observed for many other ceramics (approximate ranges given after the material), such as $\mathrm{SrTiO}_{3}(0.7-1.5 \mathrm{MRD}$ for dense ceramics and $<0.3-$ 3.7 MRD when sintered in the presence of titania-rich

liquid), ${ }^{11,27,47} \mathrm{MgAl}_{2} \mathrm{O}_{4} \quad(0.8-1.8 \mathrm{MRD}),{ }^{10,33} \mathrm{MgO}$ (0.8-2.1 MRD when lightly doped), ${ }^{12,30,48}$ or $\mathrm{TiO}_{2}$ (0.6-1.2 MRD). ${ }^{10}$ The current work used the same stereological procedure as the earlier reports, indicating that commercial-grade SOFC YSZ is significantly more isotropic than many ceramics. The GBPDs for YSZ are more similar to those determined using $2 \mathrm{D}$ data for undoped $\mathrm{MgO}$ (0.9-1.15 MRD), ${ }^{48}$ magnesia-doped alumina (0.9-1.2 MRD), ${ }^{32}$ and yttrium aluminum garnet (YAG, 0.95-1.1 MRD), ${ }^{32}$ and that determined from FIB-SEM 3D data for $\mathrm{Y}_{2} \mathrm{O}_{3}$ (0.95-1.04 MRD). ${ }^{13}$ Two points concerning the width should be discussed. First, in the case of the narrow distributions observed for YAG and $\mathrm{Y}_{2} \mathrm{O}_{3}$ (the current work used the approach from that work for collecting and analyzing data), the distributions had a decreased preference for (001) planes and an increased preference for (111) planes, ${ }^{13,32}$ that is the opposite to that observed for YSZ (or $\mathrm{MgO}^{12,30}$ and $\left.\mathrm{SrTiO}_{3}{ }^{11,47}\right)$. These differences in anisotropies indicate further that features of the true GBCD can be clearly distinguished even in fairly isotropic systems. Second, for $\mathrm{SrTiO}_{3}{ }^{11,27,47}$ and $\mathrm{MgO},{ }^{12,30,48}$ it has been observed that the width of the distributions are sensitive to processing conditions, such as if liquid phases are present during sintering and or if impurity segregation occurs. Even though the widths of the distribution were affected in those cases, the qualitative aspects of the anisotropy in the distribution were not affected. For SOFC-grade YSZ, this indicates that processing conditions generate a narrow distribution in the GBPDs, even though there is an inherent anisotropy in the GBED.

As stated in the introduction, two important observations have been made in prior works concerning the GBCD of polycrystalline materials. First, the GBCD attains a steady-state value, after which it is insensitive to further microstructural changes of scale. For tape-cast SOFC-grade YSZ, that steady-state value is already obtained after a $4-\mathrm{h}$ anneal at $1450^{\circ} \mathrm{C}$. Longer term anneals at temperatures at or below this temperature did not significantly affect the GBCD. The minor differences in the widths of the distributions for different samples is statistically insignificant. ${ }^{41}$ During the initial anneal, the particles sinter together, the compact densifies, and scale of the microstructure increases from equiaxed particles of sizes between 300 and $500 \mathrm{~nm}$ to equiaxed grains with an average size of $5 \mu \mathrm{m}$ (see Fig. 1 for distributions). Because almost no change in the microstructural scale occurs during longer term anneals at or below $1450^{\circ} \mathrm{C}$, it is unsurprising that the 
distributions also do not vary. ${ }^{28,32,35}$ During the $100 \mathrm{~h}$ anneal at $1650^{\circ} \mathrm{C}$, the scale of the microstructure increases by another order of magnitude to an average grain size of $60 \mu \mathrm{m}$. However, no change in the GBCD occurs, indicating that steady state is achieved after the initial anneal. Relative to SOFCs, this indicates that litthe thermally activated GBCD-driven microstructural evolution is expected in the single-phase electrolyte over the course of time.

The second observation is that an inverse correlation exists between the GBCD and the GBED of polycrystalline materials. That inverse correlation holds for YSZ, as demonstrated in Fig. 7. In other words, the energy (population) of grain boundaries with (111) habits is highest (lowest) while the energy (population) of grain boundaries with (100) habits is lowest (highest). Although these relative energy differences lead to rather limited anisotropies in the populations of the SOFC YSZ, the results agree with the earlier observations.

In earlier work, the anisotropies in the GBEDs of several ceramics have been rationalized by making correlations to surface characteristics of ceramic crystals. It should be clear that no specific theory that supports such correlations currently exists. ${ }^{10,13}$ In the case of $\mathrm{SrTiO}_{3}$ and $\mathrm{MgO}$, the GBED anisotropy followed the measured surface energy anisotropy. ${ }^{10}$ For YSZ, the lowest energy surface plane is generally computed to be the (111), followed by the (110), with the (100) being significantly higher in energy for the low-index surfaces (even with the inclusion of $\mathrm{Y}_{2} \mathrm{O}_{3}$ and its segregation to all surfaces). ${ }^{49-51}$ Assuming that these computational results accurately represent the surface energy anisotropy of our YSZ, our GBED anisotropies cannot be correlated with the surface energies in the same way as for $\mathrm{SrTiO}_{3}$ and $\mathrm{MgO}$; in fact, they exhibit the opposite order for the anisotropy in energy values.

In the case of $\mathrm{Y}_{2} \mathrm{O}_{3}$ and $\mathrm{MgO}$, it was further speculated that planes terminated by all cations or anions are likely to lead to high-energy grain boundaries owing to the likeliness of them being in contact with planes of mixed terminations at a grain boundary, resulting in significant increases in energy. ${ }^{13}$ Considering the arguments based on the planar composition of surfaces, the idealized (100) and (111) surfaces (outermost layers) are composed of either all anions or all cations (although consideration of multiple layers, of reconstructions, and of compositional fluctuations to the surfaces can alter the nature of the surfaces), while the (110) is composed both cations and anions. The GBED clearly indicates that boundaries having (100) habits are the lowest in energy, an observation that cannot be rationalized using correlations to ideal surface compositions (or ideal surface charges). These observations indicate that more work, especially experimental, is necessary to understand how surface energy anisotropies and GBEDs are related for YSZ in particular and polycrystals in general, as the speculations made for $\mathrm{SrTiO}_{3}, \mathrm{MgO}, \mathrm{TiO}_{2}$, and $\mathrm{Y}_{2} \mathrm{O}_{3}$, as well as for $\mathrm{Al}^{10}$ and $\mathrm{Ni}^{26}$ are not supported by the observations for YSZ. Nevertheless, the inverse correlation between the GBED and the GBPD holds for YSZ, despite our inability to rationalize the GBEDs based on surface energy calculations or growth forms (as the latter typically do not favor (100) planes).

The overall microstructure of the tape-cast YSZ ceramics can be described as dense, equiaxed structures, which obtain their steady-state microstructural characteristics after a short anneal at $1450^{\circ} \mathrm{C}$. Between 4 and $100 \mathrm{~h}$ at $1450^{\circ} \mathrm{C}$ (or lower temperatures), little evolution of the microstructure occurs, likely owing to a decreased driving force relative to the available thermal energy activating diffusional processes. By increasing the temperature to $1650^{\circ} \mathrm{C}$ (for $100 \mathrm{~h}$ ), the microstructural scale increases, as expected, but no change in the GBPD is observed. It would be interesting to observe whether or not the GBED is sensitive to temperature in this range, which will be undertaken in the future, although the temperature and grain size-independent steady-state GBPD suggests that the GBED is also constant. These equiaxed, nearly isotropic distributions imply that nearly all crystallographic features will be present in SOFC cathodes and that the local properties will be dependent on the differences in the properties of specific crystallographic features, something that is lacking in specific information from both theoretical and experimental points of view.

It has been suggested that the small anisotropies observed in the GBED and GBPD for $\mathrm{Y}_{2} \mathrm{O}_{3}$ produce the equiaxed grain structures observed in that system, ${ }^{13}$ for microstructures evolving by normal grain growth. A similar situation can be proposed for YSZ, which has equiaxed grain structures, evolves seemingly by normal grain growth, and has mild anisotropies in the GBED and GBPD. In addition, the low anisotropy is expected to yield homogeneous distributions of impurities segregating to the different boundaries and lead to homogeneous sintering events, all of which are good for achieving fully dense and homogeneous YSZ electrolytes. The situation is expected to be slightly more com- 
plex in the three-phase cathode and anode in SOFCs, and the effects of second and third phases, as well the effects of electrochemical forces, on the individual GBCDs for each phase, including YSZ, will be investigated in the future.

\section{Conclusions}

The effects of time and temperature on the GBCD of tape-cast, dense YSZ having equiaxed grain shapes were minimal, indicating that the steady-state distributions were achieved after the initial sintering step at $1450^{\circ} \mathrm{C}$ for $4 \mathrm{~h}$. During the sintering step, the average grain size grew from $<500 \mathrm{~nm}$ to $\approx 5 \mu \mathrm{m}$. A further increase in scale to a grain size $\approx 60 \mu \mathrm{m}$ did not lead to a significant change in the GBPD. No orientation or misorientation texture was observed, but mild anisotropy was observed in the grain-boundary plane distributions, with (100) orientations having larger relative areas than expected in a random distribution and (111) orientations having smaller relative areas. Similar results were found from a 3D data set collected using FIB-SEM serial sectioning for the AS sample. The GBED determined from the $3 \mathrm{D}$ data set was observed to have an inverse correlation with the GBPD. While this correlation is similar to reports for other polycrystalline materials, it was not correlated well with the surface energy anisotropies reported elsewhere. In general, these observations indicate that the processes used in SOFC production lead to uniform distributions of grain sizes and nearly random distributions in the GBCD for YSZ.

\section{Acknowledgement}

The authors thank Sukbin Lee for alignment and visualization of the $3 \mathrm{D}$ data and Katayun Barmak for useful discussion.

\section{References}

1. P. Singh and N. Q. Minh, "Solid Oxide Fuel Cells: Technology Status," Int. J. Appl. Ceram. Technol., 1 [1] 5-15 (2004).

2. N. Q. Minh and T. Takahashi, Science and Technology of Ceramic Fuel Cells, Elsevier, New York, 1995.

3. J. Fleig, "Solid Oxide Fuel Cell Cathodes: Polarization Mechanisms and Modeling of the Electrochemical Performance," Annu. Rev. Mater. Res., 33 361-382 (2003).

4. S. J. Jiang, "Development of Lanthanum Strontium Manganite Perovskite Cathode Materials of Solid Oxide Fuel Cells: A Review," J. Mater. Sci., 43 6799-6833 (2008)
5. J. R. Wilson, et al., "Three-Dimensional Reconstruction of a Solid-Oxide Fuel-Cell Anode," Nat. Mater., 5 541-544 (2006).

6. J. R. Wilson, et al., "Three-Dimensional Analysis of Solid Oxide Fuel Cell Ni-YSZ Anode Interconnectivity," Microsc. Microanal., 15 71-77 (2009).

7. D. Gostovic, et al., "Three-Dimensional Reconstruction of Porous LSCF Cathodes," Electrochem. Solid-State Lett., 10 [12] B214-B217 (2007).

8. J. R. Smith, et al., "Evaluation of the Relationship Between Cathode Microstructure and Electrochemical Behavior for SOFCs," Solid State Ionics, 180 90-98 (2009).

9. G. S. Rohrer, et al., "The Distribution of Internal Interfaces in Polycrystals," Zeitschr. Metall., 95 197-214 (2004).

10. D. M. Saylor, et al., "Habits of Grains in Dense Polycrystalline Solids," J. Am. Ceram. Soc., 87 [4] 724-726 (2004).

11. D. M. Saylor, et al., "Distribution of Grain Boundaries in $\mathrm{SrTiO}_{3}$ as a Function of Five Macroscopic Parameters," J. Am. Ceram. Soc., 87 [4] 670-676 (2004).

12. D. M. Saylor, A. Morawiec, and G. S. Rohrer, "Distribution and Energies of Grain Boundaries in Magnesia as a Function of Five Degrees of Freedom," J. Am. Ceram. Soc., 85 [12] 3081-3083 (2002).

13. S. J. Dillon and G. S. Rohrer, "Characterization of the Grain-Boundary Character and Energy Distributions of Yttria Using Automated Serial Sectioning and EBSD in the FIB," J. Am. Ceram. Soc., 92 [7] 1580-1585 (2009).

14. S. J. Dillon and G. S. Rohrer, "Three-Dimensional FIB-OIM of Ceramic Materials," Ceram. Trans., 201 117-124 (2009).

15. G. Palumbo, E. M. Lehockey, and P. Lin, "Applications for Grain Boundary Engineered Materials," JOM 50 [2] 40-43 (1998).

16. F.-S. Chen and A. H. King, "The Misorientation Dependence of Diffusion Induced Grain Boundary Migration," Scr. Metall., 20 1401-1404 (1986).

17. A. Goyal, et al., "Conductors with Controlled Grain Boundaries: An Approach to the Next Generation, High Temperature Superconducting Wire," J. Mater. Res., 12 [11] 2924-2940 (1997).

18. D. T. Verebelyi, et al., "Low Angle Grain Boundary Transport in $\mathrm{YBa}_{2} \mathrm{Cu}_{3} \mathrm{O}_{7-\delta}$ Coated Conductors," Appl. Phys. Lett., 76 [13] 1755-1757 (2000).

19. T. Nakagawa, et al., "Oxygen Diffusion Blocking of Single Grain Boundary in Yttria-Doped Zirconia Bicrystals," J. Mater. Sci., 40 3185-3190 (2005).

20. R. De Souza, et al., "Oxygen Diffusion in Nanocrystalline Yttria-Stabilized Zirconia: The Effect of Grain Boundaries," Phys. Chem. Chem. Phys. 10 [15] 2067-2072 (2008).

21. E. M. Lehockey, et al., "On Improving the Corrosion and Growth Resistance of Positive Pb-Acid Battery Grids by Grain Boundary Engineering," J. Power Sources, 78 79-83 (1999).

22. J. L. Giocondi and G. S. Rohrer, "Structure Sensitivity of Photochemical Oxidation and Reduction Reactions on $\mathrm{SrTiO}_{3}$ Surfaces," J. Am. Ceram. Soc., 86 [7] 1182-1189 (2003).

23. J. L. Giocondi, P. A. Salvador, and G. S. Rohrer, "The Origin of Photochemical Anisotropy in $\mathrm{SrTiO}_{3}$," Top. Catal., 44 [4] 529-533 (2007).

24. J. Lowekamp, et al., "Anisotropic Photochemical Reactivity of Bulk $\mathrm{TiO}_{2}$ Crystals," J. Phys. Chem. B, 102 [38] 7323-7327 (1998).

25. A. D. Rollett, et al., "Three-Dimensional Characterization of Microstructure by Electon Back-Scatter Diffraction," Annu. Rev. Mater. Res., 37 627-658 (2007).

26. J. Li, S. J. Dillon, and G. S. Rohrer, "Relative Grain Boundary Area and Energy Distributions in Nickel," Acta Mater., 57 4304-4311 (2009).

27. T. Sano, C. -S. Kim, and G. S. Rohrer, "Shape Evolution of $\mathrm{SrTiO}_{3}$ Crystals During Coarsening in a Titania-Rich Liquid," J. Am. Ceram. Soc., 88 [4] 993-996 (2005).

28. G. S. Rohrer, "Influence of Interface Anisotropy on Grain Growth and Coarsening," Annu. Rev. Mater. Res., 25 99-126 (2005).

29. J. Gruber, et al., "Effect of Anisotropic Grain Boundary Properties on Grain Boundary Plane Distributions During Grain Growth," Scr. Mater., 53 351355 (2005)

30. D. M. Saylor, A. Morawiec, and G. S. Rohrer, "The Relative Free Energies of Grain Boundaries in Magnesia as a Function of Five Macroscopic Parameters," Acta Mater., 51 3675-3686 (2003).

31. D. M. Saylor, et al., "Misorientation Dependence of the Grain Boundary Energy in Magnesia," Interface Sci., 8 131-140 (2000).

32. S. Dillon and G. Rohrer, "Mechanism for the Development of Anisotropic Grain Boundary Character Distributions During Normal Grain Growth," Acta Mater., 57 [1] 1-7 (2009). 
33. H. Miller, et al., "Crystallographic Distribution of Internal Interfaces in Spinel Polycrystals,” Mater. Sci. Forum, 467-470 783-788 (2004).

34. M. Upmanyu, et al., "Boundary Mobility and Energy Anisotropy Effects on Microstructural Evolution During Grain Growth," Interface Sci., 10 201-216 (2002).

35. E. A. Holm, G. N. Hassold, and M. A. Miodownik, "On Misorientation Distribution Evolution During Anisotropic Grain Growth,” Acta Mater., 49 2981-2991 (2001).

36. G. S. Rohrer, Structure and Bonding in Crystalline Materials, Cambridge University Press, 2004.

37. Y.-I. Yoshizawa and T. Sakuma, "Evolution of Microstructure and Grain Growth in $\mathrm{ZrO}_{2}-\mathrm{Y}_{2} \mathrm{O}_{3}$ Alloys," ISIJ Int., 29 [9] 746-752 (1989).

38. M. Boaro, J. M. Vohs, and R. J. Gorte, "Synthesis of Highly Porous YttriaStabilized Zirconia by Tape-Casting Methods," J. Am. Ceram. Soc., 86 [3] 395-400 (2003).

39. K. H. Zuo, Y. -P. Zeng, and D. Jiang, "Properties of Microstructure-Controllable Porous Yttria-Stabilized Zirconia Ceramics Fabricated by Freeze Casting," Int. J. Appl. Ceram. Technol., 5 [2] 198-203 (2008).

40. R. E. Mistler and E. R. Twiname, Tape Casting: Theory and Practice, The American Ceramic Society, Westerville, 2000.

41. H. Miller, "Influences of Processing and Composition on the Grain Boundary Character Distribution,” Ph.D., Carnegie Mellon University, Pittsburgh, 2008.

42. S. I. Wright and R. J. Larsen, "Extracting Twins From Orientation Imaging Microscopy Scan Data,” J. Microsc., 205 245-252 (2002).

43. TSL Inc. Orientation Imaging Microscopy Software Version 4.3 User Manual, EDAX, Mahwah, NJ, 2006.

44. A. Morawiec, "Method to Calculate the Grain Boundary Energy Distribution Over the Space of Macroscopic Boundary Parameter from the Geometry of Triple Junctions," Acta Mater., 48 3525-3532 (2000).

45. J. K. Mackenzie, "Second Paper on Statistics Associated with the Random Disorientation of Cubes," Biometrika, 45 [1/2] 229-240 (1958).

46. J. K. Mackenzie and M. J. Thomson, "Some Statistics Associated with the Random Disorientation of Cubes," Biometrika, 44 [1/2] 205-210 (1957).
47. T. Sano, D. M. Saylor, and G. S. Rohrer, "Surface Energy Anisotropy of $\mathrm{SrTiO}_{3}$ at $1400^{\circ} \mathrm{C}$ in Air," J. Am. Ceram. Soc., 86 [11] 1933-1939 (2003).

48. F. Papillon, G. Rohrer, and P. Wynblatt, "Effect of Segregating Impurities on the Grain-Boundary Character Distribution of Magnesium Oxide," J. Am. Ceram. Soc., 92 [12] 3044-3051 (2009).

49. S. Gennard, F. Cora, and C. R. A. Catlow, "Comparison of the Bulk and Surface Properties of Ceria and Zirconia by Ab Initio Investigations," J. Phys. Chem. B, 103 [46] 10158-10170 (1999).

50. G. Ballabio, et al., "Ab Initio of Yttria-Stabilized Cubic Zirconia Surfaces," Phys. Rev. B, 70 075417-1-075417-6 (2004).

51. X. Xia, R. Oldman, and R. Catlow, "Computational Modeling Study of Bulk and Surface of Yttria-Stabilized Cubic Zirconia," Chem. Mater., 21 [15] 3576-3585 (2009).

\section{Supporting Information}

Additional Supporting Information may be found in the online version of this article:

\section{Appendix S1. Detailed Experimental Procedure.}

Please note: Wiley-Blackwell are not responsible for the content or functionality of any supporting materials supplied by the authors. Any queries (other than missing material) should be directed to the corresponding author for the article. 